\title{
Exploring Goal-setting, Rewards, Self-monitoring, and Sharing to Motivate Physical Activity
}

\author{
Sean A. Munson \\ School of Information, University of Michigan \\ Ann Arbor, Michigan, United States \\ samunson@umich.edu
}

\begin{abstract}
Many people have turned to technological tools to help them be physically active. To better understand how goal-setting, rewards, self-monitoring, and sharing can encourage physical activity, we designed a mobile phone application and deployed it in a four-week field study $(n=23)$. Participants found it beneficial to have secondary and primary weekly goals and to receive nonjudgmental reminders. However, participants had problems with some features that are commonly used in practice and suggested in the literature. For example, trophies and ribbons failed to motivate most participants, which raises questions about how such rewards should be designed. A feature to post updates to a subset of their Facebook NewsFeed created some benefits, but barriers remained for most participants.
\end{abstract}

Keywords-Exercise; mobile applications; goal-setting; reminders; rewards; sharing; social networks; persuasive technology

\section{INTRODUCTION}

Tools such as mobile devices and social software can help people manage their health and wellness. Many applications allow people to use their computers or mobile phones to track symptoms (e.g. MAHI [20], Mobile Heart Health [22], GI Monitor, AsthmaMD), record food consumption and/or physical activity (e.g., LoseIt, UbiFit [6], Fish'n'Steps [17]), and to connect with others about their health problems, activities, and goals (e.g., PatientsLikeMe, Houston [4], SparkPeople). Online communities can also help people to gain encouragement and motivation, information, and a sense of not being alone [14,24].

We investigate four strategies to encourage physical activity through two versions of a mobile phone application that we developed, GoalPost and GoalLine, which use:

1. Goal-setting. The user is encouraged to set up to two goals each week: primary and/or secondary. If one becomes unattainable, the other may still be realistic, or, if $\mathrm{s} / \mathrm{he}$ is having a good week, s/he may push his/herself.

2. Rewards. The user receives ribbons as s/he makes progress toward goals and trophies as s/he completes them. A trophy case shows four weeks of accomplishments.

3. Self-monitoring. The user is reminded to set goals and journal activities. A "badge" icon provides a persistent reminder of the number of days since $\mathrm{s} /$ he last exercised.

4. Sharing. With GoalPost (not GoalLine), the user can share his or her goals, goal progress, and physical activities performed with his or her Facebook network.

Though these strategies have been suggested by previous work or used in commercial applications, their effectiveness is not

\author{
Sunny Consolvo \\ Human Centered Design \& Engineering, The Information School, \\ University of Washington, Seattle, Washington \\ sunny@consolvo.org
}

well understood. Klasnja et al argued that a key contribution that HCI research can make to health behavior change is a better understanding of users' experiences with behavior change strategies that are embedded in systems [16]. To that end, we used GoalPost and GoalLine as research probes to investigate how people would respond to the strategies. We deployed the applications to 23 participants from the general public in a four-week field study. Among our contributions are:

- Goal-setting. Users reported benefits from being able to concurrently pursue both primary and secondary goals.

- Rewards. Despite similarity to the badges feature of many commercial applications, the literal, expected rewards we used did not motivate, raising questions about this feature.

- Self-monitoring. The reminder notifications were one of the most appreciated features of the application.

- Sharing. Despite implementing a recommended feature from similar work - the support group - many barriers remained to sharing, though users who tried it reported some benefits.

\section{RELATED WORK}

Many applications help people initiate and maintain physical activity habits. In this brief review of related work, we focus on four promising approaches: goal-setting, rewards, selfmonitoring, and sharing.

Goal-Setting. Goals have been shown to be most effective when they are important to the individual (e.g., self-set rather than assigned), when they are realistic, when the individual can see his/her progress, and when s/he receives positive feedback as progress toward his or her goal is made. [18]. Many applications have used goal-setting as a strategy to encourage physical activity. Houston, a mobile phone application to motivate walking, provided users with a daily step count goal based on their walking history [4]. Users of UbiFit, a mobile phone application to motivate physical activity, set their own primary and alternate weekly physical goals, then chose to work toward their primary or alternate goal each week $[3,6]$. Loselt users set a long-term goal - a target weight and time frame - and then work toward daily goals that account for calories burned and consumed. These applications support pursuit of only one goal at a time. This may cause people to give up for the duration of the goal period if that goal becomes unachievable, or to avoid setting high or stretch goals to ensure that they can achieve the one goal that they do set [18].

Rewards. Many applications provide small, visual rewards for achieving milestones or completing goals. Houston provided a 
congratulatory message and a simple symbol ('*') when users met their goals. Gasser et al's healthy lifestyle coach [10] displayed emoticons $(\odot)$ or $\odot)$ according to whether a user met goals. UbiFit generated an image of a garden (a flower for each activity performed and a butterfly upon goal attainment) as the phone's wallpaper to reflect the week's activities and goal progress $[3,6]$. FitBit users receive a virtual flower that grows to indicate their recent activity level and badges for achieving goals and step count milestones. FifFu users earn awards and points. While these rewards vary in prominence and persistence, nearly all are earned at predetermined goal milestones.

Self-Monitoring and Reminders. When combined with goalsetting, performance feedback, and review of goals, selfmonitoring has been shown to be an effective component of interventions to promote physical activity [21]. Whether working toward a self-set goal, an assigned goal, or simply reflecting on one's activities, many applications include features for recording and viewing activity-related data. For example, LoseIt allows people to record their food intake and exercise on their phones. This makes journaling convenient and integrates well into daily life. Houston tracked toward a step count goal based on data users entered into their phones after reading the step counts from a pedometer [4], while UbiFit combined automated tracking with self-journaling of physical activities [3].

Other systems have focused on the potential of mobile phones to deliver specific and timely reminders. Prestwich found that subjects who received text message reminders of their plans for how to complete their goals (e.g. "don't forget to go to the gym after lecture today") increased their physical activity, while general reminders (e.g., "I don't want coronary heart disease-exercise more") or no reminders had no effect on subjects' exercise level [25]. Participants in [13] received tailored exercise solutions, weekly exercise planning, and reminders of the plan on their computers and phones; they exercised two hours more per week than those not receiving this support. UbiFit participants appreciated a feature that asked if had anything to add to their journal after two days of inactivity and the garden image on their phone's background screen that was an ever-present, gentle reminder of participants' activity level $[3,6]$. This past work shows that at least some types of reminders, especially when combined with journaling, can help people self-monitor and adjust their physical activity.

Sharing. Several systems help users share physical activityrelated data and have shown improvements in activity levels and retention rates over individual-use applications. For example, participants in an eight-week Internet-mediated physical activity program at the University of Michigan were more likely to meet their weekly physical activity goals if they joined a competitive team than if they participated as individuals [1]. In other studies, sharing physical activity levels, such as step count, has helped to motivate people to be more active through social support and social pressure. In addition to providing users with individual feedback, Houston facilitated the sharing of step counts and physical activity-related messages among a small group of friends [4]. Participants in the study's sharing condition were more likely to achieve their daily goals than participants without this feature. The Fish'n'Steps study found that sharing with strangers is not always motivating and is sometimes awkward [17]. Nevertheless, interacting with strangers can have benefits. In a 16-week Internet-mediated walking program, subjects with access to a discussion board had a $13 \%$ higher retention rate compared to a group without this feature; however, daily step count was not affected [27,28].

In addition to applications that directly connect users, many others, such as Daily Mile, RunKeeper, FitFu, Nike Fit, Adidas miCoach, FitBit, and LoseIt' connect users to their existing social networks on sites such as Twitter and Facebook. These applications typically generate suggested posts and associated data, such as maps of runs or calories burned, which users can share as status updates. Sharing on Facebook can reach friends and family whose opinions matter, but who may not be participating in the wellness activity themselves, potentially creating an additional channel for receiving social support and pressure beyond what is available by sharing only with other users of the application $[15,23,24,30]$. While previous work has argued for the potential benefits of integrating wellness activities into social network sites, it has also identified several obstacles and challenges. People are often concerned with boring their friends with mundane posts or appearing boastful about modest achievements [23,24]. Facebook networks are often quite diverse, and it may not be appropriate to share health-related data with one's entire network, even if it would be beneficial to share with a subset of that network. There are also mixed predictions for the effectiveness of sharing goals. Some work predicts that people are more likely to achieve their goals when they publicly commit to them $[12,18,29]$, while other work cautions that sharing goals with others can deliver many of the same social rewards as actually achieving the goal, and this can compromise performance [11].

In our work, we build on this previous work to further explore how to motivate physical activity using the strategies of goal-setting, rewards, self-monitoring, and sharing. We apply successful strategies and recommendations from previous work combined with variations on other features.

\section{GOALPOST AND GOALLINE}

We developed two versions of an iOS applicationGoalPost and GoalLine - that support setting weekly physical activity goals, journaling physical activity, receiving virtual rewards, and reviewing past progress. GoalPost, but not GoalLine, also facilitates sharing with the user's Facebook NewsFeed. The design of the applications was informed by the work mentioned above and an initial design survey of a convenience sample $(n=55)$ of our friends, family, and colleagues. This survey solicited feedback on ways and levels of specificity for configuring goals, on rewards, and on options for the content of NewsFeed updates that could be shared from the application.

Goal-Setting. In GoalPost and GoalLine, users set goals for a calendar week-i.e., Sunday through Saturday. Goals are broken down by category - cardio, strength, flexibility, walking, and other-to promote diversifying physical activities, viewing progress at the category level, helping users find activities in the activity list, and allowing them to specify goals at the category or specific activity level (i.e., 90 min of cardio versus 30 min of running and 60 min of elliptical). They can pick from a list of pre-defined activities or create their own. Goals need not include all five categories. Users can set "Primary" and "Secondary" goals, which they might set as a main and a backup 

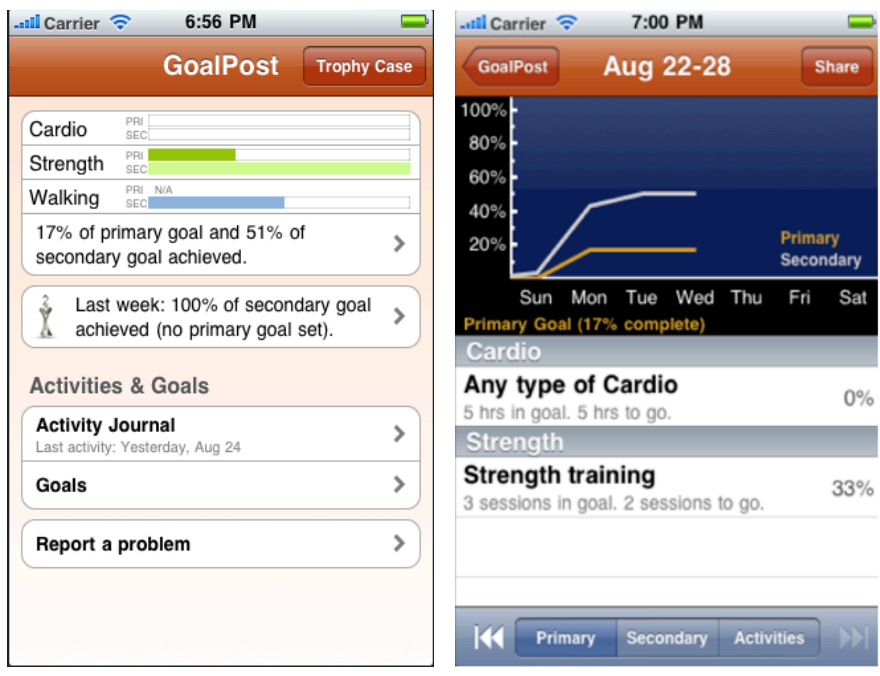

goal or a main and a stretch goal. If one goal becomes unattainable, the other may still be realistic, or if the user is having a good week, s/he may be encouraged to push his/herself. Users set one goal at a time. To set a goal, the user selects an activity, enters the target quantity for the week ("minutes" for cardio; "sessions" for strength, flexibility, and other; and either "minutes" or "steps" for walking), and then repeats for additional activities. Goals from the previous week can be used as a starting point. Users can set or change goals at any time.

Rewards. We implemented rewards earned by making progress toward and meeting goals, styling them after badges used in many commercially available applications. A "Trophy Case" (Fig 1, right-center) shows trophies and ribbons representing completed goals and activity categories for the last four weeks. Users receive a ribbon for each category within their goal that they complete (blue for categories in the primary goal, red for the secondary) - e.g., once they complete the "cardio" portion of their goal for the week, they earn a ribbon-and they receive a trophy (gold for primary, silver for secondary) if they complete all parts of their goal. Survey respondents viewed the trophies favorably: "Nice trophies. The trophy thing is way cool" and "receiving badges that got pushed to Facebook would be a nice side incentive to complete goals."

Self-Monitoring and Reminders. Users keep a physical activity journal in which they can record any activity, whether or not it counts toward a goal. The journal interface is similar to the goal-setting interface: the user navigates to the day, taps to add an item, selects the activity, sets the quantity, whether it should count toward the primary and/or secondary goals, and saves it.

Reminders. GoalPost and GoalLine provide the user with prompts (or "pop-up reminders") to journal physical activities and set goals, as well as a persistent reminder (or "notification badge") on the application's icon of how many days it has been since $\mathrm{s} /$ he last performed a physical activity.

Pop-up reminders. After a certain number of days without adding an activity to the journal, the user receives a notification stating, "It has been $n$ days since you last updated your physical activity journal." By default, these notifications begin appearing daily at 8pm on the second consecutive day without a journal update. The user can disable these reminders or change after how many days of inactivity they begin appearing and the


Figure 1. GoalPost application. Left: Goal Post's main screen with goal progress bars for different activity categories. Left-center: Viewing progress for the week. Right-center: The trophy case provided a view of the last four weeks of goal achievement. Right: Reminder "badge" on the application icon, indicating two days since the user's last recorded physical activity.

time of day at which they appear. Similar reminders appear if the user has not set a goal for the week.

Notification badge. After two days with no journal entries, a "badge" appears on the application's icon reflecting the number of days since the last recorded activity (serving as a persistent reminder; Fig 1, right). This badge cannot be disabled or otherwise configured.

Viewing Progress. GoalPost and GoalLine support selfmonitoring in a variety of ways. From the main screen (Fig 1, left), bars show progress for each of the secondary and primary goals' activity categories, as well as a percent complete for this week's and last week's overall goals. From the Goals screen, users can view the percent complete for each goal. The Goal Progress View (Fig 1, left-center), accessible from the main screen, includes a line chart that shows progress toward the user's primary and secondary goals over the week with a table of their goal items and activities; the user can navigate to previous weeks' data to view progress over time.

Sharing. GoalPost facilitates posting physical activity-related updates to the user's Facebook NewsFeed. The user can share his or her activity journal for a day or week, a single activity, a goal(s), progress toward the goal(s), or the trophy case. The user can share whenever s/he makes an update in GoalPost, as well as from most screens via a "Share" button (e.g, upper right of Figure 1, left-center \& right-center). When a user chooses to share, GoalPost provides suggestions on what to post. For example, a journal entry of 45 minutes on the elliptical trainer would suggest "journaled 45 minutes of elliptical," "journaled elliptical", or "updated his/her physical activity journal."

For goal completion, post suggestions range from neutral to positive, even when the user falls short of his or her goal(s) (e.g., GoalPost suggests the percent completed, rather than that the user has "failed" to meet the goal). This follows recommendations to be positive and not punish users [23] and addresses concerns from design survey respondents about negative posts: "Most people don't meet their goals. I wouldn't want a listing of everyone's shortcomings on my Facebook feed." $88 \%$ of respondents from our design survey said that they would not want to post if they were running far behind their goal, one calling such a post "horrendous."

Privacy. Some users might have concerns about sharing their physical activity data with Facebook or with members of their 
Facebook network. To prevent accidental sharing, posting is a multi-step process that can be cancelled at any point: users must press share, select a post (which could be edited), select with whom the post will be shared and whether it should include a link off of Facebook (described below), and then press another button to complete sharing the post.

To address concerns about sharing with Facebook the company, GoalPost allows users to include a link to more details available off of Facebook while leaving the post vague. This way, detailed information is available to the user's friends but is not stored on Facebook's servers or with the user's other Facebook data. Responses in our design survey were mixed, though some found it important: "I like people knowing the details but I do NOT like Facebook knowing the details." Another potential benefit of this feature that others pointed out is that it lets friends decide what level of detail they want to see.

One nice thing about the linked one is that way you're only sharing details with people who are interested enough to click the link, instead of automatically sharing with everyone.

Previous work has also found that Facebook networks can be too broad for people to feel comfortable sharing health information [24]. A user might benefit from sharing with some of his or her friends, while fearing that s/he may bore others with over-sharing or be misunderstood or taunted. Many survey respondents expressed a desire to share with only a subset of their network: "friends - great! 'friends' - leave me alone" and "If teammates, fine, if general Facebook friends, not very interesting." To provide some control, GoalPost includes a support group feature that allows users to send updates to a subset of their Facebook network. The list is administered on a website, and GoalPost users can decide on a per-post basis if they want to share with their support group or their entire network. To limit the chances of a user sharing more broadly than intended, GoalPost defaults to sharing only with the support group. If a user posts to their support group without having created one, only that user can see the post.

\section{GOALPOST AND GOALLINE FIELD STUDY}

23 people participated in an exploratory field study that included four weeks with either GoalPost (with sharing) or GoalLine (no sharing); see Table 1. A market research agency recruited participants from the general public in the Seattle metropolitan area who had an active Facebook account, owned an iPhone $3 \mathrm{G}$ or more recent version, were between 20 and 50 years old, and were in the Contemplation, Preparation, or Action stage of change for physical activity according to the Transtheoretical Model [26].

Participants began to use the application on a Thursday or Friday at a session at the researchers' offices that included informed consent, installation of the app on their personal iPhone, an introduction to the application, a background questionnaire, and measures of their height and weight (used to calculate BMI). Participants used the application for slightly more than four weeks, including four full Sunday through Saturday goal periods, during which they completed five online surveys about their experiences. These surveys asked about any problems with the application, successes, and if they had talked about their goals or physical activity with anyone. The first survey also asked additional questions about setting goals, the
TABLE I. PARTICIPANT INFORMATION.

\begin{tabular}{llllll}
\hline ID & Gender & Age & Stage of Change & BMI & BMI Category \\
\hline GP1 & F & 29 & Contemplation & 44.6 & obesity class iii \\
\hline GP2 & M & 21 & Action & 21.4 & normal \\
\hline GP3 & M & 24 & Preparation & 27.1 & overweight \\
\hline GP4 & M & 37 & Contemplation & 29.0 & overweight \\
\hline GP5 & M & 38 & Preparation & 29.7 & overweight \\
\hline GP6 & F & 36 & Action & 32.7 & obesity class i \\
\hline GP7 & F & 39 & Contemplation & 23.5 & normal \\
\hline GP8 & F & 33 & Preparation & 35.4 & obesity class ii \\
\hline GP9 & M & 49 & Action & 26.7 & overweight \\
\hline GP10 & F & 46 & Preparation & 32.3 & obesity class i \\
\hline GP11 & F & 28 & Action & 24.1 & normal \\
\hline GP12 & M & 29 & Preparation & 23.1 & normal \\
\hline GL13 & F & 48 & Preparation & 26.8 & overweight \\
\hline GL14 & M & 26 & Contemplation & 23.0 & normal \\
\hline GL15 & M & 44 & Action & 22.5 & normal \\
\hline GL16 & M & 32 & Preparation & 31.0 & obesity class i \\
\hline GL17 & F & 34 & Contemplation & 21.4 & normal \\
\hline GL18 & F & 20 & Action & 30.9 & obesity class i \\
\hline GL19 & F & 34 & Action & 23.2 & normal \\
\hline GL20 & F & 29 & Preparation & 24.7 & normal \\
\hline GL21 & M & 26 & Action & 37.7 & obesity class ii \\
\hline GL22 & M & 36 & Preparation & 40.7 & obesity class iii \\
\hline GL23 & M & 40 & Preparation & 30.6 & obesity class i \\
\hline
\end{tabular}

second about journaling, the third about reviewing progress, and the fourth about changing goals.

After the field use, participants returned for individual semi-structured interviews about their experiences and to receive compensation for participating. The $23 \mathrm{~h} 42 \mathrm{~min}$ of interview data (ave: $1 \mathrm{~h} 02 \mathrm{~m}$, range: $36 \mathrm{~m}$ to $1 \mathrm{~h} 31 \mathrm{~m}$ ) were recorded and transcribed. Their survey responses helped tailor the semistructured interviews. Researchers coded the interview transcripts and open-ended responses from the surveys. Responses were grouped based on our initial research questions as well as reactions to particular and potential features. Additional categories were made for recurring themes.

\section{RESUlTS}

Overall, participants valued the application; 17 elected to continue using it ${ }^{1}$ after the study. Participants reported that the application reminded them of their activity level, pushed them to incrementally increase their exercise, reminded them to exercise in busy weeks, and encouraged them to add variety to their exercise routine. The purpose of this study was to investigate particular strategies and their implementations to encourage physical activity, as advocated for by [16], and to compare the outcomes (goals, goal achievement, and activities journaled) between participants who used GoalPost and GoalLine. However, limited use of the sharing feature in GoalPost meant that we were unable to make meaningful comparisons between the two conditions. Thus, for this section, we review results across GoalPost and GoalLine participants as they relate to goal-setting, rewards, self-monitoring, and sharing.

Goal-setting. On average, each participant set 3.8 primary goals and 2.3 secondary goals during the four full weeks of the study. Participants achieved $58 \%$ of each primary goal (meeting the goals $22 \%$ of the time) and $49 \%$ of each secondary goal

\footnotetext{
${ }^{1}$ This was 17 out of 21 participants; technical limitations prevented us from offering this option to two of the 23 participants.
} 
(meeting them $14 \%$ of the time). Participants generally found the goals easy and straightforward to set. Some reported enjoying making the commitment to themselves, e.g., "just simply entering the goals-there was something about, 'I've got to swim twice, 'that was kind of a charge" (GP9).

Secondary and Primary Goals. 19 participants set both secondary and primary goals for at least one of the four weeks. These 19 most commonly used one as their routine goal (i.e., what they believed they normally did) ${ }^{2}$ and the other as their stretch goal (i.e., a real challenge) (10 participants), a routine goal and something that would be nice to do or add variety (4 participants), a routine goal and a backup goal (3 participants), and a stretch goal and a backup goal (2 participants). Another participant was unsure what each goal meant to her. ${ }^{3}$ Of these 19,14 would likely continue using secondary and primary goals regularly; two would use both sometimes, such as on weeks when they were unsure how much time they would have for exercise; two would not continue using both types of goals; and one did not specify a preference. Most who had both a routine or backup goal and a stretch goal said that the stretch goal motivated them to go above and beyond what they would otherwise have done. They described feeling less committed to their stretch or "nice to do" goal (GP12, GL16) and not feeling guilty if they did not meet it (GP12, GL14), but that not meeting their routine or backup goal felt like a "failure" (GL14).

Rewards. Though the survey we used to inform our application design suggested that people would be motivated by virtual trophies and ribbons for achieving goals and portions of their goals, the field study participants had mixed reactions to this feature. The trophy and ribbon rewards only seemed to motivate three participants (GP1, GP7, and GL23), two of whom were in the contemplation phase and one in the preparation phase of the Transtheoretical Model. GP7 considered lowering her goal mid-week "because I really wanted the trophy thing," and described not getting one as "disappointing." GP1 was less strongly motivated: "it was just kind of that little satisfaction of like-I have a little gold trophy." GP12 thought the rewards would be motivating until he experienced his first trophy, and then stopped caring. Though GL22 did not find them to be particularly motivating, she did like that they provided a quick way to remind her, "I accomplished one thing at least" and helped her look back on her accomplishments. Most others were apathetic about the literal rewards-describing them as not particularly motivating, nor a nuisance or bother. Some of the apathy resulted from the trophies never being a surprise (GP8, GL19). When participants journaled an activity, they already knew that they had met the goal or portion of the goal, so receiving the trophy or ribbon simply did not tell them new information: "It really didn't do anything for me. Because I knew I'd met my goals when I entered it and I was like okay, turn it off" (GP8). Six participants thought the trophies and ribbons were "lame" (GP2), an "unnecessary" reward (GP3, GP5, GL16), or a "gimmick" (GP9, GL20), though only two of these six ever earned a trophy. None of the participants had another reward system in place for meeting their goals.

\footnotetext{
${ }^{2}$ During the study, many participants came to realize that they did less physical activity than they thought they had been doing.

${ }^{3}$ This totals to 20 because one participant alternated between using the two goals as routine/nice to do and routine/stretch.
}

Self-monitoring and reminders. Participants thought the activity journal was simple and quick to use and that entering their activity data was often a "reward" (GL13), not a burden. Most appreciated that the journal feature was "casual" (GP1) and convenient, especially because it was on their phone ("it's always with you... you're just like, 'Ba ba ba, done." (GL14)). Several participants wanted to be able to journal additional details (e.g., repetitions, distance, or pace); a few wanted to use these criteria when specifying goals. Despite finding journaling helpful, many participants said that they doubted they would have kept up with journaling without reminders. Overall, they found both types of reminders to be effective at helping them stick to their exercise goals, but the badges frustrated some.

Pop-up reminders. The pop-up reminders were a particularly well-liked feature. When asked what they liked, the reminders were the first feature mentioned by 15 participants, and there was no evidence of reminder fatigue over the study. None of the participants disabled the reminder feature, and one set them to be more frequent. Participants described the reminders as gentle and not overly nagging, in contrast to reminders that might come from someone they knew, such as a parent:

I think it was kind of a nice realization in terms of what I am and am not doing and it was, you know, without having to have, you know, sort of mom or someone else involved going, "Oh, you haven't been exercising." (GP1)

One participant, however, noted that it was "almost like my mom" (GP4). The reminders were sometimes considered "annoying" or "a nuisance" but this was seen as good:

it was annoying, but that's why I liked it. ...there's always that voice in the back of your head just saying, "Stay on the couch. Relax. Eat those potato chips." And so that [the pop-up reminder] was kind of like the angel on the shoulder telling you to go do something. (GP3)

"It was a love-hate relationship but, yeah, it was good for me" (GP8).

Participants felt good when they received a reminder and had an activity that they had forgotten to journal, while reminders received when they did not have an activity to journal tended to cause slightly negative feelings. These reminders increased participants' awareness of their activity levels and helped them to remember their commitment to themselves:

I liked that the app reminded me because it's so easy to get busy and just forget that you had intentions of exercising and then the week's suddenly over and you were like oh yeah, I wanted to do that. (GP5)

Reminders sometimes spurred participants to be more active immediately, such as by getting up and going for a walk.

Notification badge. Reactions to the notification badge were mixed. For some, this badge competed with their mental model of how iOS badges work. Some participants thought that they should be able to dismiss the badge by opening the application. However, with GoalPost/GoalLine, the badge indicated the number of days since the most recent physical activity; journaling an activity was the only way to dismiss the badge. Some participants were frustrated that they could not just open the application to make the badge "go away" (GP11, GP12, GL15, GL19). Other participants, though, found their need to dismiss the badge motivating enough to exercise (GP8, GL21).

Sharing. Ten of the 12 GoalPost participants used the sharing feature at least once (Table 2). However, this number is somewhat misleading; four shared with an empty support group, making them the only person on Facebook who could see it, 
and a fifth (GP1) shared by accident. Some of the posts to an empty support group were intentional, but most were the result of forgetting that they had not set up a group. Only three configured and used a support group. None included a link off of Facebook to more detailed information in their posts.

Participants named many barriers to sharing. The most prevalent concern, echoing previous work [23, 24], was a fear of appearing boring or boastful about something trivial.

It's just kind of embarrassing to me... I jogged for 20 minutes today, let me tell 200 people, many of which I haven't talked to in years ... Who cares?... I don't want to share with someone that I, you know, they comment on it, oh yeah I ran a marathon, good to go 20 minute runner guy. (GP12)

The support group feature mitigated these concerns for some participants. GP7 set up a support group with just one friend, rather than sharing with her entire network:

Because she's accepting and she wouldn't get sick of my posts. I guess when we were talking about this and choosing a support group, all I could think of was that Farmville thing where I'm always seeing that someone has a cow. That is so annoying. I didn't want to annoy another person.

Other participants also echoed the need for a support group feature, as their Facebook networks were "too broad" and had people who they knew these types of posts would bother or people who "didn't need to know" their physical activity goals and accomplishments. GP1 was concerned that sharing would set unrealistic expectations:

\begin{abstract}
it's, you know, one of those start and fail kind of things and so I don't want anyone to even know it's happening and maybe they'll just be pleasantly surprised in a couple months and go, "Hey, you look like you maybe lost a little weight or something." ... rather than, "Oh weren't you on a diet?"
\end{abstract}

Despite these barriers, those who shared-even infrequently_-saw some benefits from this sharing. GP7's sister-in-law, her one support group member, commented about seeing the post while on the phone and also "liked" the post on Facebook, which felt like "somebody's cheering for me." Some felt that sharing increased their accountability, while others felt better knowing that the commitment was just for them and not shared. GP2 found an exercise partner as a result of sharing his activity on Facebook through GoalPost, which resulted in him going to the gym more frequently than he used to. Another participant shared only with an empty support group, but still found that seeing his post on his NewsFeed was motivating:

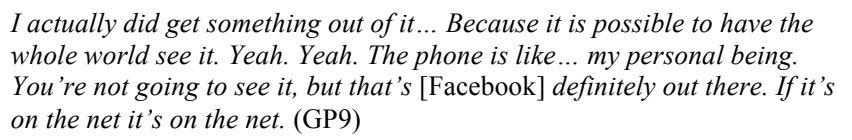

Another said that she "really enjoyed being able to post what I did," but wished that the posts had gotten more of a reaction from the people in her support group:



GP11 thought that she would have benefited more from posting what was still left to do, since those posts might have helped her find activity partners. GP4 also posted once, but did
TABLE II. SHARING SUMMARY

$\begin{array}{lllll} & \end{array}$

not get a reaction, and so decided not to post again, believing that this meant that his friends were not interested:

It comes down to needing reaction. If I post things that no one reacts to, there's a reason they're not reacting and I don't want to annoy them. (GP4)

GP7 was also disappointed by the sharing feature:

GP7: I'm really depressed. If Facebook can't save me, I don't know what will help me lose weight.

Researcher: Did you think Facebook was going to help?

GP 7: I thought it was going to save me, yeah.

Researcher: Did it?

GP7: No. And I'm so discouraged.

Her disappointment was partly about a lack of reaction, but also because she felt that she did not have enough successes to share. Like GP11, she thought that it might be better if GoalPost automatically posted what she had left to do: "Now there's the shame factor of "[I] didn't do anything".... I think that would help. Shame's always a good healthy thing." However, this speculation about preferring to automatically share failures was not representative.

Though many participants were concerned with Facebook, the company, having too much of their personal data, and some had cut back on adding other information to Facebook, none felt uncomfortable sharing their GoalPost data with Facebook. They did not feel that this information needed to be protected. Some said that they would have concerns about posting other types of health data, especially body weight or running routes. They were similarly unconcerned with others finding this data on their phone. One participant, though, did not trust that support group posts would stay private, having had prior bad experiences with Facebook retroactively changing default settings.

\section{DISCUSSION}

Our field study helped explore goal-setting, rewards, selfmonitoring, and sharing in a mobile application to promote physical activity and highlights design challenges that need further investigation. We focus here on two promising strategies (the use of both secondary and primary goals and the reminders), and two strategies (rewards and sharing) for which our implementations were less compelling than anticipated.

Goal-setting: Use Secondary and Primary Goals. Most participants responded well to the use of both secondary and primary goals that were attempted simultaneously. Working to- 
ward simultaneous goals helped participants stretch beyond what they would otherwise have done and gave them a fallback during busy weeks that helped them remember to do at least some exercise. Based on the success of the implementation of this feature in our four-week field study, a logical next step is a long-term field deployment, such as in an RCT, to evaluate whether the use of multiple, simultaneous goals is simply preferred or whether it actually leads to better health behaviors.

Goal-setting and Self-monitoring: Use Reminders. Though participants found keeping a journal helpful, many doubted they would have kept it up without the reminders. Despite the positive reactions, the participants' experiences suggest further improvements and variations that should be evaluated in an RCT. One idea is to include progress toward the goal or a list of activities remaining for the week, similar to the implementation intention reminders used by [25] or Houston's reminders of how many steps remained in their daily goal [4], but at the risk of being more of a nag. Some participants speculated that the application could be just reminders, simply asking them each day whether they had exercised (GP2, GP3), though at least one participant thought that would be too passive (GP8). Another possibility, based on the principle of kairos [8], would be to combine reminders with context awareness to deliver the reminders when the user is likely to be in a position to perform an activity. Future work could also address how goal-setting affects the reminders' effectiveness. If users simply had a journal with reminders to update it, would the reminders be as effective as in an application in which users also set goals?

Issues with Literal, Expected Rewards. The prevalence of badges and other rewards in commercially available systems, and the strong, positive reaction in our design survey to earning and sharing trophies, caused us to have high expectations for the ribbons and trophies in GoalPoast/GoalLine. However, only three participants in our study described these rewards as motivating. While similar to the badges in some applications, such as FitBit, the GoalPost/GoalLine ribbons and trophies are different from rewards that have been previously shown to be successful in motivating physical activity. In UbiFit, the garden was both a reminder and a reward that the user "grew" over time, while in Fish'n'Steps, the user nurtured his or her fish, and Houston provided a simple symbol, GoalPost and GoalLine's trophies and ribbons were more literal than those rewards, and this may have contributed to them seeming like a "gimmick" or "childish" (GP10). Also, unlike UbiFit, where users got a flower for every activity performed, GoalPost/GoalLine users did not see any rewards until they completed an entire subcategory of their goal. The user also had to open the application - and thus already be thinking about physical activity - in order to see the rewards, unlike the persistent home screen in UbiFit.

The GoalPost/GoalLine rewards also behaved differently from some other successful rewards, such as badges or accomplishments in videogames or applications such as FourSquare and Yelp. In those applications, people may know that an award is possible to achieve, but not the exact criteria, and earning a badge is often a surprise that offers new information. These factors have been found to be important for delivering effective verbal feedback [7] and other persuasive applications (e.g., [9]) but were not characteristics of the trophies and ribbons in
GoalPost/GoalLine. In contrast, expected rewards were motivating in the Houston and UbiFit systems. Future work should address this contradiction to better understand what characteristics of rewards - literal or abstract, expected or surprising, at what point rewards should be provided - are motivating for which personalities and behaviors.

Sharing: Being interesting and getting support. Sharing routine physical activity information on Facebook was not particularly compelling for any of the GoalPost participants. Even though we implemented a support group feature to allow targeting posts to particular friends, as recommended but unimplemented in previous research [23,24], GoalPost participants still had concerns about sharing too often or too broadly. The hesitation to share, and consequences of over-sharing, may also prevent users from experiencing the predicted benefits of sharing. Another issue that participants encountered was that when they did share, they seldom received the support they wanted or expected. Given these issues, is sharing with one's existing online social network a strategy worth pursuing? We still believe that it is. Similar features are both prevalent and used in commercial applications, and GoalPost participants who tried it reported some benefits. Achieving benefits, such as receiving social support or finding activity partners, with fewer barriers or negative consequences, will require further investigation.

What to share. Posts could be more interesting by including photos from hikes or running routes, or reflecting bigger milestones such as running a marathon or achieving a weight loss goal. "You're going to post something, it should catch people's attention, because you're using up their time." (GL14). GP8 felt that adding details about calories burned, repetitions, or pace would make the posts interesting enough to share. However, because some of these posts also reveal more personal information-e.g., a route might reveal a home address-some participants expressed reservations about sharing too broadly.

With whom to share. Some participants said that sharing a challenge or a group goal with one or more of their friends, or with teammates or running group members might make them feel more comfortable sharing. Common themes included feeling a need for reciprocity or going through the experience together and for knowing that the people they were sharing with were in a similar position. In contrast to [17] but consistent with [4], several participants thought that they would benefit more from sharing with an accountability partner who was a stranger but whose activity level, fitness, and goals were similar than with friends who are in a very different situation. The importance of both types of similarity-characteristics related and unrelated to performance - has been discussed extensively in social comparison literature (e.g., [31]). Explicitly inviting friends to one's support group could also help. In many support groups, members join because they also need support or are asked to participate; simply posting updates about activity to one's Facebook network may not make a user's needs clear.

Study Limitations This was a study to learn about participants' experiences with various motivational strategies in an effort to inform the design of applications to support physical activity. As such, it had a limited number of participants and no baseline data, so we are unable to meaningfully measure change in activity. Regarding reminders and the use of second- 
ary and primary goals, future work should investigate their effectiveness with larger numbers of participants over longer durations, while further exploratory work is required for the reward and sharing features. Also, some participants were worried that stopping sharing at the conclusion of the study would appear like a failure; they were also concerned that their friends could not download and use the application. To address these concerns, future studies should consider allowing continued use of the application after the study's conclusion, and researchers may wish to make the application available to members of participants' social networks.

\section{CONCLUSION}

In this paper, we described the GoalPost and GoalLine research probes that we developed to investigate motivating physical activity using goal-setting, rewards, self-monitoring, and sharing. In a four-week field study, we found that the reminders and goal-setting strategies in GoalPost and GoalLine were appealing. Our successes with reminders and with the use of secondary and primary goals should be followed up with long-term field studies. The literal rewards of trophies and ribbons were less motivating than expected, despite the prevalence of similar features in other systems, and we suggest possible reasons that should be investigated in future work. Posting to one's Facebook network created some benefits, but figuring out with whom and what to share was still problematic and did not get participants the support they desired and expected, despite our implementation of recommendations from previous work. The lessons learned from our study can inform the design of applications to promote physical activity or other behavior change, and highlight needs for future research.

\section{ACKNOWLEDGEMENTS}

The authors conducted this study at Intel Labs Seattle. Adidas and Jared Jones contributed to the design of GoalPost/GoalLine. Thank you to the Michigan Interactive and Social Computing group and anonymous reviewers for feedback.

\section{REFERENCES}

[1] Buis LR, Poulton TA, Holleman RG, Sen A, Resnick PJ, Goodrich DE, Palma-Davis L, Richardson C. Evaluating Active U: an internetmediated physical activity program. BMC Public Health 2009;9:331.

[2] Consolvo S, Everitt K, Smith I, Landay JA. Design Requirements for technologies that encourage physical activity. CHI 2006.

[3] Consolvo S, Klasnja P, McDonald DW, Avrahami D, Froehlich J, LeGrand L, Libby R, Mosher K, Landay JA. Flowers or a robot army?: encouraging awareness \& activity with personal, mobile displays. UbiComp 2008: 54-63.

[4] Consolvo S, Klasnja P, McDonald DW, Landay. JA. Goal-Setting Considerations for Persuasive Technologies that Encourage Physical Activity. Persuasive 2009.

[5] Consolvo S, McDonald DW, Landay JA. Theory-Driven Design Strategies for Technologies that Support Behavior Change in Everyday Life. CHI 2009.

[6] Consolvo S, McDonald DW, Toscos T, Chen MY, Froehlich J, Harrison B, Klasnja P, LaMarca A, LeGrand L, Libby R, Smith I, Landay JA. Activity Sensing in the Wild: A Field Trial of UbiFit Garden. CHI 2008.

[7] Deci EL, Kostner R, Ryan RM. A Meta-Analytic Review of Experiments Examining the Effects of Extrinsic Rewards on Intrinsic Motivation. Psychol Bull 1999;125(6): 627-668.

[8] Fogg BJ. Persuasive Technology: Using Computers to Change What We Think and Do. Morgan Kaufmann 2003.
[9] Froehlich J, Consolvo S, Dillahunt T, Harrison B, Klasnja P, Mankoff J, Landay JA. UbiGreen: Investigating a Mobile Tool for Tracking and Supporting Green Transportation Habits. CHI 2009.

[10] Gasser R, Brodbeck D, Degen M, Luthiger J, Wyss R, Reichlin S. Persuasiveness of a Mobile Lifestyle Coaching Application Using Social Facilitation. Persuasive 2006: $27-38$.

[11] Gollwitzer PM, Sheeran P, Michalski V, Seifert AE. When Intentions Go Public: Does Social Reality Widen the Intention-Behavior Gap? Psychol Sci 2009;20(5): 612-618.

[12] Hollenbeck FR, Williams CR, Klein HJ. An empirical examination of the antecedents of commitment to difficult goals. J Appl Psychol 1989;74: 18-23.

[13] Hurling R, Catt M, De Boni M, Fairley BW, Hurst T, Murray P, Richardson A, Sodhi JS. Using Internet and Mobile Phone Technology to Deliver an Automated Physical Activity Program: Randomized Controlled Trial. J Med Internet Res 2007;9(2):e7.

[14] Hwang KO, Ottenbacher AJ, Green AP, Cannon-Diehl MR, Richardson O, Bernstam EV, Thomas EJ. Social support in an Internet weight loss community. IJMI 2010;79(1): 5-13.

[15] Khaled R, Barr P, Noble J, Biddle R. Investigating Social Software as Persuasive Technology. Persuasive 2006.

[16] Klasnja P, Consolvo S, Pratt W. How to Evaluate Technologies for Health Behavior Change in HCI Research. CHI 2011.

[17] Lin JJ, Mamykina L, Lindtner S, Delajoux G, Strub HB. Fish'n'Steps: Encouraging Physical Activity with an Interactive Computer Game. Ubicomp 2006.

[18] Locke EA, Latham GP. Building a Practically Useful Theory of Goal Setting and Task Motivation: A 35-Year Odyssey. American Psychologist, 2002;57(9): 705-17.

[19] Locke EA, Latham GP. Goal Setting Theory. In Smith KG, Hitt MA (eds.). Great minds in management: the process of theory development. 2005: $128-150$

[20] Mamykina L, Mynatt E, Davidson P, Greenblatt, D. MAHI: Investigation of Social Scaffolding for Reflective Thinking in Diabetes Management, CHI 2008: 477-86.

[21] Michie S, Abraham C, Whittington C, McAteer J, Gupta S. Effective Techniques in Healthy Eating and Physical Activity Interventions: A Meta-Regression. Health Psychol 2009; 28(6): 690-701.

[22] Morris ME, Guilak F. Mobile Heart Health: Project Highlight. IEEE Pervasive 2009;8(2): 57-61.

[23] Munson SA, Lauterbach D, Newman MW, Resnick P. Happier Together: Integrating a Wellness Application Into a Social Network Site. Persuasive 2010

[24] Newman MW, Lauterbach D, Munson S, Resnick P. It's not that I don't have problems, I'm just not putting them on Facebook": Challenges and Opportunities in Using Online Social Networks for Health. CSCW 2011.

[25] Prestwich A, Perugini M, Hurling R. Can the effects of implementation intentions on exercise be enhanced using text messages? Psychol Health 2009;24(6): 677-687.

[26] Prochaska JO, Velicer WF. Transtheoretical model of health behavior change. Am J Health Promot 1997;12(1): 38-48.

[27] Resnick PJ, Janney AW, Buis LR, Richardson CR. Adding an Online Community to an Internet-Mediated Walking Program. Part 2: Strategies for Encouraging Community Participation. J Med Internet Res 2010;12(4):e72.

[28] Richardson CR, Buis LR, Janney AW, Goodrich DE, Sen A, Hess ML, Mehari KS, Fortlage LA, Resnick PJ, Zikmund-Fisher BJ, Strecher VJ, Piette JD. An Online Community Improves Adherence in an InternetMediated Walking Program. Part 1: Results of a Randomized Controlled Trial. J Med Internet Res 2010;12(4):e71.

[29] Salancik G. Commitment and the control of organizational behavior and belief. In Staw BM, Salancik GR (eds.). New directions in organizational behavior. 1977: 1-54.

[30] Skeels MM, Unruh KT, Powell C, Pratt W. Catalyzing social support for breast cancer patients. $\mathrm{CHI} 2010$ : 173-182

[31] Wood JV. Theory and Research Concerning Social Comparisons of Personal Attributes. Psychol Bull 1989;106(2): 231-248. 